\title{
ON THE PTÁK HOMOMORPHISM THEOREM
}

\author{
B. RODRIGUES
}

(Received 13 May, 1988; revised 19 September 1988)

Communicated by S. Yamamuro

\begin{abstract}
In this note, a brief and accessible proof is given of an extension of the Pták homomorphism theorem to a larger class of spaces-spaces that are not necessarily assumed to be locally convex. This is done by first proving a counterpart of the Bourbaki-Grothendieck homomophism theorem for the non-locally-convex case. Our presentation utilizes the simplifying properties of seminorms.

1980 Mathematics subject classification (Amer. Math. Soc.) (1985 Revision): 46 A 30, 47 A 05. $K e y w o r d s$ and phrases: semi-barrelled space, barrelled space, semi- $B$-complete, $B$-complete, quotient seminorm, adequate map, small adjoint, semi-open, weakly open, nearly semi-open, nearly semi-continuous.
\end{abstract}

\section{Introduction}

This note originates from a study of Köthe's generalization [5] of the Pták homomorphism theorem where the extension given is obtained in the case the spaces are locally convex. What we do here is to extend these results to the non-locally-convex situation (and therefore to a larger class of spaces) in a simple and direct way. This is done by first proving a non-locallyconvex analog (see Lemma 2) of what we call the Bourbaki-Grothendieck homomorphism theorem (see Theorem 4). This result, which is significant in itself, then allows us to derive the more general theorem (see Theorem 10). Our extensions reduce to those of Köthe when the spaces under consideration are locally convex. The locally-convex theory can also be found in [1], [2], [3], [4], [7], [10] and [11].

(C) 1989 Australian Mathematical Society 0263-6115/89 \$A2.00+0.00 
In order to achieve our results, we first extend, in Section 2, the definitions for $B$-complete and barrelled spaces from Pták theory to the non-locallyconvex situation where we define semi-B-complete and semi-barrelled spaces. In Section 3, we develop, with the aid of seminorms, a duality theory which allows us to give a characterization of what we call a nearly semi-open map and to derive our main results. In Section 4, we extend results obtained in Section 3, to weakly open maps and discuss what we call nearly semicontinuous maps.

Apart from obtaining more general results, our proofs are much less complicated than those given in [5] and [7] where the arbitrary linear case is first reduced to the continuous one. Here, our use of duality arguments provides a simplification of Köthe's results and a direct approach to Pták's theorem.

In order to keep our treatment short and simple, we do not consider the question of maximal mappings as is done in [5] and [7]. Here, every map is defined on the entire domain space and hence assumed to be maximal (see [7, Section 36.7(2), page 91]).

\section{Preliminaries}

Let $E$ and $F$ be real Hausdorff topological vector spaces and $T: E \rightarrow F$ be a linear (but not necessarily continuous) map. If $P$ is a seminorm defined on $E$, we define the quotient seminorm, $P / T$, on (the image of $T) T(E)$, by

$$
P / T(y):=\inf P\left(T^{-1} y\right) \quad(y \in T(E)) .
$$

Remark. The quotient seminorm $P / T$ can be defined alternatively by

$$
P / T(T x):=\inf \{P(x+\operatorname{Ker} T)\} \quad(x \in E) .
$$

We say that $T$ is semi-open if $P / T$ is continuous on $T(E)$ whenever $P$ is continuous on $E$.

The following are basic definitions (see [6, Section 10.7, page 90], [7, Section 32, page 1]: $T$ is open if it is an open map from $E$ onto $T(E)$ (where $T(E)$ has the topology induced from $F$ ) and is weakly open if it is open when $E$ and $F$ are equipped with the weak topologies $\sigma\left(E, E^{\prime}\right)$ and $\sigma\left(F, F^{\prime}\right)$. We say that $T$ is weakly continuous if it is continuous from $\left(E, \sigma\left(E, E^{\prime}\right)\right)$ into $\left(F, \sigma\left(F, F^{\prime}\right)\right.$ ). If $T$ is (weakly continuous) continuous and (weakly open) open then it is a topological (weak homomorphism) homomorphism.

We note that in the case both $E$ and $F$ are locally convex, $T$ is semi-open if and only if $T$ is open. This follows directly from the identity

$$
\{y: y \in T(E), P / T(y)<1\}=T\{x: x \in E, P(x)<1\} .
$$


Also, since $\left(E, \sigma\left(E, E^{\prime}\right)\right)$ and $T(E)$ under the topology induced from $\sigma\left(F, F^{\prime}\right)$ (which we will denote by $\left.\left.\sigma\left(F, F^{\prime}\right)\right|_{T(E)}\right)$ are locally convex, we have the following equivalent definition of a weakly-open map: $T$ is weakly open if $P / T$ is continuous on $\left(T(E),\left.\sigma\left(F, F^{\prime}\right)\right|_{T(E)}\right)$ whenever $P$ is continuous on $\left(E, \sigma\left(E, E^{\prime}\right)\right)$. In the case where only $F$ is locally convex, we have the following example of semi-open maps:

EXAMPLE. If $E$ is any topological vector space and $F$ is locally convex, then any open map is semi-open.

If $E^{\prime}$ and $E^{*}$ denote the topological dual and the algebraic dual of $E$ respectively, define $E_{P}^{\prime}$ and $E_{P}^{*}$ by

$$
E_{P}^{\prime}:=\left\{a: a \in E^{\prime}, a \leq P \text { on } E\right\}
$$

and

$$
E_{P}^{*}:=\left\{a: a \in E^{*}, a \leq P \text { on } E\right\} .
$$

We now define the small adjoint of $T, T^{t}: \Delta \rightarrow E^{\prime}$, by $T^{t} d:=d \circ T(d \in \Delta)$ where $\Delta:=\left\{d: d \in \overline{T(E)}^{\prime}, d \circ T \in E^{\prime}\right\}$ (here $\overline{T(E)}$ has the topology induced from $F$ ) and say that $T$ is adequate if for all $x \in E$ and for all $a \in E^{\prime}$,

$$
\left\langle x, T^{t}(\Delta)\right\rangle=\{0\} \text { and }\langle\operatorname{Ker} T, a\rangle=\{0\} \text { implies }\langle x, a\rangle=0 .
$$

REMARKS (ON THE SMALL ADJOINT). If for all $d \in \Delta$, there exists $d^{\prime} \in F^{\prime}$ such that $\left.d^{\prime}\right|_{\overline{T(E)}}=d$, then $T^{t}(\Delta)=\left\{d \circ T: d \in F^{\prime}, d \circ T \in E^{\prime}\right\}=: T^{\prime}(D)$ (the range of the adjoint of $T$ ) where $D:=\left\{d: d \in F^{\prime}, d \circ T \in E^{\prime}\right\}$ (the domain of the adjoint). In particular, this is the case when $F$ is locally convex. It is this distinction that leads us to sue the term "small adjoint".

REMARKS (ON ADEQUATE MAPS). It is clear that $T$ is adequate if $\Delta$ separates points of $T(E)$ or if $\overline{\operatorname{Ker} T}=E$. In particular, $T$ is adequate if $F$ is locally convex and $T$ is continuous.

EXAMPLE. The canonical injection map $l^{1 / 2} \hookrightarrow l^{1}$ (see [11, 1.1, page 2] for a definition of $\left.l^{p}(0<p<1)\right)$ has dense range in $l^{1}$ and hence is adequate since, in this case, $\Delta=l^{1}=l^{\infty}$ (or otherwise, since $l^{1}$ is locally convex and the injection map is continuous).

Also, we define $E$ to be semi-B-complete if every subspace $L$ of $E^{\prime}$ is $\sigma\left(E^{\prime}, E\right)$-closed in $E^{\prime}$ whenever $L \cap E_{P}^{*}$ is $\sigma\left(E^{\prime}, E\right)$-compact for every continuous seminorm $P$ on $E$. We do not assume that $E$ is locally convex. In the case $E$ is locally convex, the notion of a semi- $B$-complete space is equivalent to that of a $B$-complete ( $\equiv$ Pták) space. 
EXAMPLes. Any topological vector space with a degenerate dual is semi$B$-complete. For example, the $L^{p}(0<p<1)$ (see $[11,1.1$, page 2] for a definition) spaces are semi- $B$-complete. Also, (for the same reason) $L^{2}(0,1)$ with the topology induced from $L^{1 / 2}(0,1)$ is semi- $B$-complete. The latter example provides us with a semi- $B$-complete space which is not complete. Indeed, this is clear since $L^{2}(0,1)$ is dense in the complete space $L^{1 / 2}(0,1)$ (see [4, 5.7(D), page 98]). Compare this with the fact that every $B$-complete space is necessarily complete

We say that $F$ is semi-barrelled if every lower-semicontinuous seminorm on $F$ is continuous. Here, again, we do not assume local convexity, requiring only that for $F$ to be semi-barrelled, every barrel is a neighbourhood of zero. We do not assume that $F$ has a zero-neighbourhood base of barrels. In the case $F$ is locally convex, then semi-barrelled is equivalent to barrelled (in the usual sense). Compare the definitions given here with [6, Section 21.2(2)-(3), page 257] and [9, Section 5, page 249] and note also that if, in the sense of [9], $F$ is ultrabarrelled, then it is semi-barrelled in our terminology, although the converse is not necessarily true, even in the locally convex case, as the following example, given by $W$. Robertson in [9, Section 7 , page 256], shows: $l^{1 / 2}$ with the norm topology $\left(\|\cdot\|_{1}\right)$ induced from $l^{1}$ is semi-barrelled (barrelled) but not ultrabarrelled. This example also shows that, for the locally-convex situation, in contrast with the concept of semi-barrelledness, ultrabarrelledness is not equivalent to that of barrelledness.

Before we give an example of a non-locally-convex and semi-barrelled space which is not ultrabarrelled, we have the following

EXAMPLes. Any Baire topological vector space is semi-barrelled. Let $E$ be Baire and $B$ be a barrel in $E$. From standard proofs (see [10, II.7.1, page 60] for example), $B$ has some interior point $x$. Since $B$ is balanced, $0=$ $1 / 2 x+1 / 2(-x)$ is interior to $B$ and hence $B$ is a neighbourhood of zero. In particular, the complete metrizable $l^{p}(0<p<1)$ spaces are semi-barrelled. More generally, any $F$-space (complete metrizable topological vector space) is semi-barrelled. Note that we already know that a Baire space is ultrabarrelled (see [9, Section 5, Proposition 12]).

ReMARKS (ON THE ASSOCIATED LOCALlY-CONVEX TOPOLOGY). Suppose that $E$ has topology $\mathscr{I}$; then the topology $\mathscr{T}^{00}$ (our notation follows that in [9, Section 7, page 255]), formed by taking as its zero-neighbourhood base the convex and balanced $\mathscr{T}$-neighbourhoods of zero, is a locally-convex topology coarser than $\mathscr{T}$. This topology, which is called the associated locally-convex topology, is the finest locally-convex topology coarser than $\mathscr{T}$ and is Hausdorff if $\left(E, E^{\prime}\right)$ is a dual pair, where $E^{\prime}=(E, \mathscr{T})^{\prime}$. Moreover, $\left(E, \mathscr{T}^{00}\right)^{\prime}=E^{\prime}$ 
(see $[9$, Section 7 , page 255]), a fact we will use in the next example. In that we already know if $E$ is ultrabarrelled under $\mathscr{T}$ then $E$ is barrelled under $\mathscr{T}^{00}$ (see [9, Section 7, Proposition 19]), we can show, more generally, that if $E$ is semi-barrelled under $\mathscr{T}$ then it is barrelled under $\mathscr{T}^{00}$ : let $B$ be a $\mathscr{T}^{00}$-barrel; then $B$ is a $\mathscr{T}$-barrel (since $\mathscr{T}^{00} \subset \mathscr{T}$ ) and hence a (convex and balanced) $\mathscr{T}$-neighbourhood of zero. This proves that $B$ is a $\mathscr{T}^{00}$-neighbourhood of zero.

We can now give an example of a semi-barrelled space which is not ultrabarrelled. Here, our notation follows that in [11].

EXAMple. Consider the identity map from $\left(l^{1 / 2},\|\cdot\|_{1 / 2}\right) \rightarrow\left(l^{1 / 2},\|\cdot\|_{3 / 4}\right)$ where $\|\cdot\|_{1 / 2}$ and $\|\cdot\|_{3 / 4}$ denote the usual topologies of $l^{1 / 2}$ and $l^{3 / 4}$ respectively. Since $\|\cdot\|_{3 / 4}$ is coarser than $\|\cdot\|_{1 / 2}$, the identity is continuous but not open. Since $\left(l^{1 / 2},\|\cdot\|_{1 / 2}\right)$ is complete metrizable, an application of $W$. Robertson's open mapping theorem ([9, Section 6 , page 252]) to the identity shows that $\left(l^{1 / 2},\|\cdot\|_{3 / 4}\right)$ is not ultrabarrelled. On the other hand $\left(l^{1 / 2},\|\cdot\|_{3 / 4}\right)$ is semibarrelled: from the above examples, $\left(l^{1 / 2},\|\cdot\|_{1 / 2}\right)$ is semi-barrelled. Let $B$ be a $\|\cdot\|_{3 / 4}$ barrel; then, since $\|\cdot\|_{3 / 4}$ is coarser than $\|\cdot\|_{1 / 2}$ and $\left(l^{1 / 2},\|\cdot\|_{1 / 2}\right)$ is semibarrelled, $B$ is a $\|\cdot\|_{1 / 2}$-neighbourhood of zero. To show that $B$ is a $\|\cdot\|_{3 / 4^{-}}$ neighbourhood of zero, it suffices to show that it is a $\|\cdot\|_{1}$-neighbourhood of zero (since $\|\cdot\|_{1} \subset\|\cdot\|_{3 / 4}$ ). We need to show therefore that each convex and balanced $\|\cdot\|_{1 / 2}$-neighbourhood of zero is a $\|\cdot\|_{1}$-neighbourhood of zero. We do this by proving that $\|\cdot\|_{1}=\mathscr{T}^{00}$ where $\mathscr{T}^{00}$ is the locally-convex topology associated with $\|\cdot\|_{1 / 2}$. We first show that $\|\cdot\|_{1} \supset \mathscr{T}^{00}$. From $[9$, Section 7 , page 256] we know that $\left(l^{1 / 2},\|\cdot\|_{1}\right)^{\prime}=l^{\infty}$ so $\|\cdot\|_{1}=\tau\left(l^{1 / 2}, l^{\infty}\right)$ (the Mackey topology). Hence we need only show that $\left(l^{1 / 2},\|\cdot\|_{1}\right)^{\prime}=\left(l^{1 / 2}, \mathscr{T}^{00}\right)^{\prime}$ or, equivalently, that $\left(l^{1 / 2},\|\cdot\|_{1}\right)^{\prime}=\left(l^{1 / 2},\|\cdot\|_{1 / 2}\right)^{\prime}$ (see "Remarks (on the associated locally-convex topology)" above). But this is immediate since $\left(l^{1 / 2},\|\cdot\|_{1 / 2}\right)^{\prime}=$ $l^{\infty}$ (see $\left[11,9.3, \# 123\right.$, page 140]). On the other hand, since $\mathscr{T}^{00}$ is the finest locally-convex topology coarser than $\|\cdot\|_{1 / 2}$, we have $\|\cdot\|_{1} \subset \mathscr{T}^{00}$.

REMARK (ON THE BANACh-Steinhaus TheOREM). Standard proofs of this theorem for the locally-convex case (see [4, 11.1, pages 219-221], [10, III.4.2, page 83] for example) are easily adapted to the non-locally-convex situation to obtain the following: for $E$ semi-barrelled and $F$ locally convex, every simply (pointwise) bounded set $H \subset \mathscr{L}(E, F)$ is equicontinuous. In particular, this is valid for $E$ semi-barrelled and $F=\mathbf{R}$ or, as we have seen, since every Baire space is semi-barrelled, when $E$ is a Baire space and $F$ locally convex.

In the following sections, $E$ and $F$ are real Hausdorff topological vector spaces and $T$ is assumed to be adequate unless otherwise stated. 


\section{The main results}

LEMMA 1. $b \in T(E)_{P / T}^{*}$ if and only if $b \circ T \in E_{P}^{*}$.

Proof. (i) For all $x \in E,\langle x, b \circ T\rangle=\langle T x, b\rangle \leq P / T(T x)=\inf P\left(T^{-1} T x\right)$ $\leq P(x)$

(ii) For all $y \in T(E)$ and $x \in T^{-1} y,\langle y, b\rangle=\langle T x, b\rangle=\langle x, b \circ T\rangle \leq P(x)$. Taking the infimum over $x$ gives $\langle y, b\rangle \leq P / T(y)$.

LEMMA 2. Let $T^{t}(\Delta)$ be $\sigma\left(E^{\prime}, E\right)$-closed and assume that

for every continuous seminorm $P$ on $E$, there exists a continuous seminorm $Q$ on $T(E)$ such that, for $b \in T(E)^{\prime}, b \circ T \in E_{P}^{\prime}$ implies $b \in T(E)_{Q}^{\prime}$.

Then $T$ is semi-open.

Proof. We first show that $b \in T(E)^{*}$ and $b \circ T \in E^{\prime}$ implies $b \in T(E)^{\prime}$. Suppose that $x \in E$ and $\left\langle x, T^{t}(\Delta)\right\rangle=\{0\}$. Since $T$ is adequate and $\langle\operatorname{Ker} T, b \circ$ $T\rangle=0,\langle x, b \circ T\rangle=0$. It follows by the assumption that $T^{t}(\Delta)$ is $\sigma\left(E^{\prime}, E\right)$ closed and the separation theorem that $b \circ T \in T^{t}(\Delta)$. This implies there exists a $d \in \Delta$ such that $T^{t} d=b \circ T$ and hence that $b=\left.d\right|_{T(E)} \in T(E)^{\prime}$.

Now, for $b \in T(E)_{P / T}^{*}$, by Lemma $1, b \circ T \in E_{P}^{*} \subset E^{\prime}$ and therefore, from the above, $b \in T(E)^{\prime}$. Since (by the Hahn-Banach theorem) $P / T$ is the supremum of linear functionals it dominates, we then have $P / T=$ $\sup \left\{b: b \in T(E)_{P / T}^{\prime}\right\}$. By Lemma 1 and assumption (3), this implies that

$$
P / T=\sup \left\{b: b \in T(E)^{\prime}, b \circ T \in E_{P}^{\prime}\right\} \leq \sup \left\{b: b \in T(E)_{Q}^{\prime}\right\} \leq Q
$$

for some continuous seminorm $Q$. This shows that $P / T$ is continuous and hence that $T$ is semi-open.

Lemma 2 is fundamental to this note and significant in its own right. It not only will allow us to derive an extension to the Pták homomorphism theorem (see Theorem 10) but also throws light on, and parallels in the nonlocally-convex situation, the Bourbaki-Grothendieck homomorphism theorem (Köthe, [7, Section 32.4(3), page 8], attributes this theorem to Grothendieck; we also find it in Bourbaki [1, IV, Section 4, Proposition 2]). We state this theorem next for comparison and easy reference. In what follows, $\mathfrak{M}_{E}$ and $\mathfrak{M}_{F}$ denote the classes of equicontinuous subsets in $E^{\prime}$ and $F^{\prime}$ respectively. 
THEOREM 4 (BOURBAKI-GROTHENDIECK HOMOMORPHISM THEOREM). Let $E$ and $F$ be locally convex and $T$ be continuous. Assume that $T^{l}\left(F^{\prime}\right)$ is $\sigma\left(E^{\prime}, E\right)$-closed and that

$$
T^{t}\left(F^{\prime}\right) \cap \mathfrak{M}_{E} \subset T^{t}\left(\mathfrak{M}_{F}\right)
$$

Then $T$ is open.

Here (5) reads as follows: every equicontinuous subset of $E^{\prime}$ that is contained in $T^{t}\left(F^{\prime}\right)$ is contained in the image under $T^{t}$ of some equicontinuous subset of $F^{\prime}$.

Condition (5) in Theorem 4 (that is, in the case $E$ and $F$ are locally convex and $T$ is continuous) is equivalent to $T$ being nearly open (see [5, Section 8(1), page 321], [7, Section 34.1, page 23]). In the case $T$ is arbitrary we have the following dual characterization of nearly-open maps between locally-convex spaces which is due to Köthe (see [5, Section 8(4), page 322], [7, Section 37.3(4), page 99]):

$$
T \text { is nearly open if and only if } T^{t}(D) \cap \mathfrak{M}_{E} \subset T^{t}\left(D \cap \mathfrak{M}_{F}\right),
$$

where $D$ denotes the domain of the adjoint.

We show now that our condition (3) in Lemma 2 is, in fact, equivalent to (6) in the locally convex situation and for arbitrary $T$.

LEMMA 7. If $E$ and $F$ are locally convex, $T$ is nearly open if and only if (3).

Proof. In view of $(6)$, it suffices to show that

$$
T^{t}(D) \cap \mathfrak{M}_{E} \subset T^{t}\left(D \cap \mathfrak{M}_{F}\right) \text { if and only if (3). }
$$

(i) Let $M_{1} \subset T^{t}(D)$ and $M_{1} \in \mathfrak{M}_{E}$. Then since equicontinuous subsets can be determined by continuous seminorms ( $H \subset E^{\prime}$ is equicontinuous if and only if there exists a continuous seminorm $P$ on $E$ such that $h \leq P$ for all $h \in H)$, we can write $M_{1}=\left\{d \circ T: d \in F^{\prime}, d \circ T \in E^{\prime}, d \circ T \leq P\right\}$ for some continuous seminorm $P$ on $E$. Since, for each $d \in F^{\prime},\left.d\right|_{T(E)} \in T(E)^{\prime}$, we deduce from (3) that $M_{1} \subset\left\{d \circ T: d \in F^{\prime}, d \circ T \in E^{\prime},\left.d\right|_{T(E)} \leq Q\right\}$ for some continuous seminorm $Q$ on $T(E)$. Since $Q$ determines an equicontinuous subset of $T(E)^{\prime}$, we find that $M_{1} \subset T^{t}(D \cap M)$ for $M \in \mathfrak{M}_{F}$ and where $\left.M\right|_{T(E)}$ is the equicontinuous set determined by $Q$ (see [4, 8.7(2), page 163]). It follows that $M_{1} \subset T^{t}\left(M_{2}\right)$ where $M_{2} \subset D$ and $M_{2} \in \mathfrak{M}_{F}$ is given by $M_{2}:=D \cap M$.

(ii) Let $P$ be a continuous seminorm on $E$. For $b \in T(E)^{\prime}$ there exists (since $F$ is locally convex, by the Hahn-Banach theorem) $b^{\prime} \in F^{\prime}$ such that $\left.b^{\prime}\right|_{T(E)}=b$. If $b \circ T \in E_{P}^{\prime}$ then $b^{\prime} \circ T \in E_{P}^{\prime}$ and hence $b^{\prime} \circ T \in\left\{d \circ T: d \in F^{\prime}\right.$, 
$\left.d \circ T \in E^{\prime}, d \circ T \leq P\right\}$. Since $P$ is continuous it determines an equicontinuous subset of $E^{\prime}$ so that we can now deduce, from the left-hand-side of (8), that

$$
\left\{d \circ T: d \in F^{\prime}, d \circ T \in E^{\prime}, d \circ T \leq P\right\} \subset T^{t}(M)
$$

for some $M \subset D$ and $M \in \mathfrak{M}_{F}$. Hence $b^{\prime} \circ T \in\left\{d \circ T: d \in F^{\prime}, d \circ T \in\right.$ $\left.E^{\prime}, d \leq Q^{\prime}\right\}$ where $Q^{\prime}$ is the continuous seminorm on $F$ which determines $M$. It follows that $b \leq Q$ where $Q:=\left.Q^{\prime}\right|_{T(E)}$ is continuous on $T(E)$.

It is also easily seen that a semi-open map satisfies (3): let $P$ be a continuous seminorm on $E$ and $b \in T(E)^{\prime}$. By Lemma $1, b \circ T \in E_{P}^{\prime}$ implies $b \in T(E)_{P / T}^{\prime}$, so (3) is satisfied by taking $Q:=P / T$.

In view of Lemma 7 and this observation, condition (3) in Lemma 2 can be taken to be an extension to the non-locally-convex situation of the dual characterization (6), for nearly-open maps and, therefore, defines what we will henceforth call a nearly semi-open map (see the example following Corollary 11).

We now make some additional remarks on adequate maps. The condition

$$
(x, 0) \in G^{00} \text { implies } x \in(\operatorname{Ker} T)^{00},
$$

where "0" denotes the operator of polarity and $G:=\{(x, T x): x \in E\}$ is the graph of $T$ ensures that $T$ is adequate: let $\left\langle x, T^{\prime}(\Delta)\right\rangle=\{0\}$ and $\langle\operatorname{Ker} T, a\rangle=$ $\{0\}$. If $(c, b) \in G^{0} \subset E^{\prime} \times F^{\prime}$ then $b \circ T=-c \in E^{\prime}$ and hence $d:=\left.b\right|_{T(E)} \in \Delta$ and $c=-d \circ T=-T^{t} d$. Hence, $\langle(x, 0),(c, b)\rangle=\langle x, c\rangle=-\left\langle x, T^{t} d\right\rangle=0$, which is to say, $\langle x, 0\rangle \in G^{00}$. By $(9), x \in(\operatorname{Ker} T)^{00}$ so $\langle x, a\rangle=0$. If, in addition, $F$ is assumed to be locally convex then (9) is equivalent to $T$ being adequate, since, in this case, the above argument can be reversed. In the case both $E$ and $F$ are locally convex, by the bipolar theorem, (9) is equivalent to $T$ being weakly singular ( $T$ is weakly singular if $(x, 0) \in \bar{G} \Rightarrow x \in \overline{\operatorname{Ker} T}$ ) (see [5, Section 2, page 310], [7, Section 36.1, page 81]).

We now have

THEOREM 10. Let $E$ be semi-B-complete and assume that $T$ is nearly semiopen. The $T$ is semi-open.

Proof. We first show that for each continuous seminorm $P$ on $E, T^{t}(\Delta) \cap$ $E_{P}^{*}$ is $\sigma\left(E^{\prime}, E\right)$-compact:

$$
T^{t}(\Delta) \cap E_{P}^{*}=\left\{b \circ T: b \in T(E)^{\prime}, b \circ T \in E_{P}^{\prime}\right\}=\left\{b \circ T: b \in T(E)_{P / T}^{\prime}\right\}
$$

by standard extension arguments and Lemma 1 . Define $R: T(E) \rightarrow \mathbb{R}$ by $R:=\sup \left\{b: b \in T(E)^{\prime}, b \circ T \in E_{P}^{\prime}\right\}$ so that $T$ is continuous, since, as $T$ is nearly semi-open, by (3), $R \leq Q$ for some $Q$ continuous on $T(E)$. Also, by Lemma $1, R=\sup \left\{b: b \in T(E)_{P / T}^{\prime}\right\}$. Hence $T(E)_{P / T}^{\prime}=T(E)_{R}^{\prime}$ which then 
implies that $T(E)_{P / T}^{\prime}$ is $\sigma\left(T(E)^{\prime}, T(E)\right)$-compact. The claim follows by the $\sigma\left(T(E)^{\prime}, T(E)\right)-\sigma\left(E^{*}, E\right)$ continuity of the map $b \mapsto b \circ T$.

Since $E$ is semi- $B$-complete, $T^{t}(\Delta)$ is $\sigma\left(E^{\prime}, E\right)$-closed and the conclusion of the theorem now follows from Lemma 2.

In view of the comparative observations given in this section, it is easily verified that Theorem 10 extends the Pták homomorphism theorem (Corollary 13) or its generalizations as given in, for example, [4, 9.7(1), page 186], [5, Section 11, page 325], [7, Section 37.5(4), page 104] and [10, IV.8.3, page 163]. In particular, in [5, Section 11, page 325] we find that $T$ is open if $E$ and $F$ are assumed to be locally convex and $T$ to be weakly singular and nearly open. It is clear that Theorem 10 reduces to this result if we take $E$ and $F$ to be locally convex, since, in this case, as we have already seen, the following are equivalent: (i) weakly singular; (ii) adequate and nearly open; (iii) nearly semi-open. We also have

COROLLARY 11. Let E be semi-B-complete and assume that $T(E)$ is semibarrelled. Then $T$ is semi-open.

Proof. The assumption that $T(E)$ is semi-barrelled satisfies condition (3) by taking, for each continuous seminorm $P$ on $E, Q$ to be the (lowersemicontinuous) seminorm defined by $Q:=\sup \left\{b: b \in T(E)_{P / T}^{\prime}\right\}$ and by using Lemma 1 . Hence $T$ is nearly semi-open and the corollary now follows from Theorem 10.

RemarK. It is clear from the proof of Corollary 11 that if $F$ is semibarrelled, then any linear map from an arbitrary topological vector space onto $F$ is nearly-semi-open (cf. [7, Section 34.1(1), page 24]).

EXAMPLE. The canonical injection map $l^{1 / 2} \hookrightarrow l^{1}$ is nearly semi-open: its image, $l^{1 / 2}$, under the norm topology induced from $l^{1}$ is semi-barrelled (as we have already seen) and consequently, by the argument given in the proof to Corollary 11 above, (3) is satisfied and the injection is nearly semi-open. It is clear, however, from the definition and the fact that the norm topology $\|\cdot\|_{1}$ is (strictly) coarser than the usual topology $\|\cdot\|_{1 / 2}$ on $l^{1 / 2}$, that the injection is not semi-open. This example also shows that a complete metrizable space need not necessarily be semi- $B$-complete since, as the injection map has already been shown to be adequate, in view of Corollary $11, l^{1 / 2}$ is not semi- $B$ complete.

Further, in view of the "Remarks (on adequate maps)" on the connection between adequate and continuous we now have the following corollaries for continuous maps. 
COROLlary 12. Let $E$ be semi-B-complete, $F$ be locally convex and assume that $T(E)$ is barrelled. Then $T$ is semi-open.

CoRollary 13 (PTÁK's homomorPhism THEOREM). Let E be B-complete (that is, a Pták space), $F$ be locally convex and barrelled and assume that $T$ is surjective. Then $T$ is open.

\section{Ancillary results}

In order to prove our next result on weakly-open maps we will use the following result from $[11,9.1, \# 104$, page 131]:

LEMMA 14. For $S \subset E^{\prime}, S$ is $\sigma\left(E, E^{\prime}\right)$-equicontinuous if and only if $S \subset$ conv $S_{1}$ for some finite subset $S_{1}$ of $E^{\prime}$ (that is, $S$ is contained in a polyhedron).

Theroem 15. Let $F$ be locally convex and $T^{t}(\Delta)\left(=T^{t}(D)\right)$ be $\sigma\left(E^{\prime}, E\right)$ closed. Then $T$ is weakly open.

Proof. We need to show that $P / T$ is continuous on $\left(T(E), \sigma\left(F, F^{\prime}\right) \mid T(E)\right)$ whenever $P$ is continuous on $\left(E, \sigma\left(E, E^{\prime}\right)\right)$. From the proof given for Lemma 2 , it is clear that it suffices to show that for every seminorm $P$ continuous on $\left(E, \sigma\left(E, E^{\prime}\right)\right)$, there exists a seminorm $Q$ continuous on $\left(T(E), \sigma\left(F, F^{\prime}\right) \mid T(E)\right)$ such that, for $b \in T(E)^{\prime}, b \circ T \in E_{P}^{\prime}$ implies $b \in T(E)_{Q}^{\prime}$ We now show this. Since $P$ is continuous on $\left(E, \sigma\left(E, E^{\prime}\right)\right)$, it determines a $\sigma\left(E, E^{\prime}\right)$-equicontinuous subset $S \subset E^{\prime}$. By Lemma 14, $S \subset \operatorname{conv} S_{2}$, where $S_{1}$ is a finite subset of $E^{\prime}$. Hence, for every $b \in T(E)^{\prime}$ such that $b \circ T \in E^{\prime}$ and $b \circ T \leq P$, $b \circ T \subset \operatorname{conv} S_{1}$. From this, it is straightforward to verify, using the linearity of the map $b \mapsto b \circ T$, that there exists a finite set $S_{2} \subset T(E)^{\prime}$ such that $b \in \operatorname{conv} S_{2}\left(\subset T(E)^{\prime}\right)$. From Lemma 14 again, conv $S_{2}$ is $\sigma\left(T(E), T(E)^{\prime}\right)$ equicontinuous and hence $b \leq Q$, where $Q$ is the $\sigma\left(T(E), T(E)^{\prime}\right)$-continuous seminorm which determines conv $S_{2}$. Since $F$ is locally convex, $Q$ is continuous in the topology on $T(E)$ induced by $\sigma\left(F, F^{\prime}\right)$; that is, $Q$ is continuous on $\left(T(E), \sigma\left(F, F^{\prime}\right) \mid T(E)\right)$.

Remarks. Theorem 14 parallels [5, Section 9(9), page 324], [7, Section 37.4(6), page 102]. Under the conditions in Theorem 14, $T$ is a weak homomorphism if, in addition, $T$ is weakly continuous.

To complete our discussion, we now briefly examine the notion of nearlycontinuous maps (see [5, Section 10, page 324], [7, Section 34.6, page 36]). To 
extend this concept to the non-locally-convex case in the context of the duality theory as developed in Section 3, we say that $T$ is nearly semi-continuous if

for every continuous seminorm $Q$ on $T(E)$, there exists a continuous seminorm $P$ on $E$ such that, for $b \in T(E)^{\prime}$ and $b \circ T \in E^{\prime}, b \in T(E)_{Q}^{\prime}$ implies $b \circ T \in E_{P}^{\prime}$.

From this definition we find that (i) if $T$ is injective then $T$ is nearly semicontinuous if and only if $T^{-1}$ is nearly semi-open, and (ii) If $E$ is semibarrelled, then every linear map from $E$ into any topological vector space is nearly semi-continuous. Both (i) and (ii) are easily verified. We give a proof only for (ii): let $E$ be semi-barrelled and $Q$ be a continuous seminorm on $T(E)$. The conclusion in (16) is then obtained by choosing $P$ to be the lowersemicontinuous seminorm defined by $P:=\sup \left\{b \circ T: b \circ T \in E^{\prime}, b \in T(E)_{Q}^{\prime}\right\}$ (cf. [5, Section 10, page 324], [7, Section 34.6, page 36]).

In this case $E$ and $F$ are locally convex, Köthe has shown [5, Section 10(2), page 325] that for $T$ arbitrary, $T$ is nearly continuous if and only if $T^{t}\left(D \cap \mathfrak{M}_{F}\right) \subset \mathfrak{M}_{E}$. Using this fact and arguments similar to those given in Lemma 7 , we can easily verify that, in the case both $E$ and $F$ are locally convex, nearly semi-continuous and nearly continuous are equivalent.

EXAMPLE. The inverse to the injection map $l^{1 / 2} \hookrightarrow l^{1}$ is nearly semicontinuous by (ii) above since $l^{1 / 2}$ under the norm topology induced from $l^{1}$ is semi-barrelled. This is also related, via (i), to the fact that we already know that the injection map itself is nearly semi-open (see the example following Corollary 11).

\section{Concluding remarks}

Although we have touched on some of the fundamental notions in Pták theory, other concepts connected with the theory can be extended along similar lines to the non-locally-convex situation. For example, it is clear how we can extend the definitions for infra-barrelled and $B_{r}$-complete spaces to give ones for "infra-semi-barrelled" and "semi- $B_{r}$-complete" respectively. This suggests the possibility that the many other results of the theory can be extended while proofs can be simplified.

In conclusion, however, we ask some questions of interest that arise from this note.

QUESTION 1. Is it possible to give an equivalent definition (similar to that given for semi-open maps) of a nearly semi-open map using quotient seminorms? 
Question 2. The "Remark (on the Banach-Steinhaus theorem)" suggests the following: is every simply bounded set $H \subset \mathscr{L}(E, F)$ equicontinuous if $E$ is semi-barrelled and $F$ an arbitrary topological vector space? (It is already known that $H$ is equicontinuous if $E$ is ultrabarrelled and $F$ an arbitrary topological vector space [9, Section 5, page 250]).

Question 3. In Theorem 15, can we drop the assumption that $F$ is locally convex?

QUESTION 4. In connection with obtaining a "closed-graph" theorem (see $[10$, IV.8.5, page 166]), is every closed subspace of a semi- $B$-complete ("semi$B_{r}$-complete") space, semi- $B$-complete ("semi- $B_{r}$-complete")?

\section{Acknowledgment}

The author would like to thank the referee and Professor S. Simons for very helpful comments.

\section{References}

[1] N. Bourbaki, Topological vector spaces, (Springer-Verlag, New York-Heidelberg-Berlin, 1987).

[2] R. E. Edwards, Functional analysis, theory and applications, (Holt Rinehart and Winston, New York, 1965).

[3] J. Horváth, Locally convex spaces, (Lecture notes in mathematics, 331, Ed. L. Waelbroeck, Springer-Verlag, New York-Heidelberg-Berlin, 1973).

[4] H. Jarchow, Locally convex spaces, (B. G. Teubner, Stuttgart, 1981).

[5] G. Köthe, 'General linear transformations of locally convex spaces', Math. Ann. 159 (1965), 309-328.

[6] G. Köthe, Topological vector spaces I, (Springer-Verlag, Berlin-Heidelberg-New York, 1969).

[7] G. Köthe, Topological vector spaces II, (Springer-Verlag, New York-Heidelberg-Berlin, 1979).

[8] V. Pták, 'Completeness and the open mapping theorem', Bull. Soc. Math. France 86 (1958), 41-74.

[9] W. Robertson, 'Completions of topological vector spaces,' Proc. London Math. Soc. 8 (1958), 242-257.

[10] H. H. Schaefer, Topological vector spaces, (Springer-Verlag, New York-Heidelberg-Berlin, 1986).

[11] A. Wilansky, Modern methods in topological vector spaces, (McGraw-Hill, New York, 1978).

\section{Department of Mathematical Sciences}

Loyola University

New Orleans, Louisiana 70118

U.S.A. 